\title{
Minimally invasive (MIE) Vs open surgery for cancer of esophagus and GE Junction. Long term results.
}

Binay Thakur ${ }^{1}$, Di Yonghui ${ }^{1}$, Mukti Devkota ${ }^{1}$, Paribartan Baral ${ }^{1}$, Yogesh Regmi ${ }^{2}$, Rajendra Malli ${ }^{3}$, Upasana Tiwari ${ }^{3}$

${ }^{1}$ Department of Surgical Oncology, BP Koirala Memorial Cancer Hospital

${ }^{2}$ Department of Anesthesiology, BP Koirala Memorial Cancer Hospital

${ }^{3}$ Department of Nursing, BP Koirala Memorial Cancer Hospital

Correspondence: Dr. Binay Thakur, Chief, Department of Surgical Oncology, BP Koirala Memorial Cancer Hospital, Bharatpur, Nepal. Phone : +9779855055931

Email: binaythakur@hotmail.com

\begin{abstract}
Introduction: MIE is becoming a preferred approach for cancer of esophagus and GE junction, but its benefits have yet to be confirmed in randomized controlled trials. We performed a retrospective comparison between these two approaches for complications and survival results.
\end{abstract}

Methods: Patients primarily going for surgery or after neoadjuvant chemo/ chemoradiation were reviewed. MIE varied from totally thoracoscopic and laparoscopic to a hybrid approach. Both MIE and Open approaches were a three, two or one incision procedures.

Results: MIE and open surgery were done in 128 and 293 patients, respectively. The mean basic parameters (referred as MIE/ Open) - age (59/ 57 years), duration of dysphagia (4/ 4 months), weight loss (8/ $9 \mathrm{~kg})$, postoperative stay (13/ 14 days), hospital mortality (3/ 17), anastomotic leak (17/32), hoarseness of voice (8/ 16), number of harvested nodes (21/22), and R0 resection (122/263) did not differ significantly ( $p=n s)$. Mean operative time (265/ 240 mins) and intraoperative blood loss (325/ $436 \mathrm{ml}$ ) differed significantly $(p<0.05)$. Postoperative pneumonia was significantly less in MIE group $(p<0.001)$. Median and 5 -year overall survival was 33 months and $21 \%$ in MIE group; and 27 months and $20 \%$ in open group $(p=0.2)$

Conclusion: MIE and Open surgery did not differ in major postoperative complications except postoperative pneumonia, which was less in MIE. Both the procedures were similar in achieving RO resection, median and 5-year overall survival.

Key words: Esophageal cancer; Mckeon's esophagectomy, VATS 